\section{Multidisciplinary Care: For the Sake of Our Patients}

\author{
Christopher K. Bichakjian, MD
}

It is difficult to imagine a field of medicine in which the importance of multidisciplinary care is greater than in oncology. Whether a patient is treated for breast cancer or sarcoma, prostate cancer or melanoma, the ensuing journey through the maze of oncologic care will undoubtedly, directly or indirectly, cross the paths of multiple specialists. Within the field of cutaneous oncology, this may range from a patient with a large basal cell carcinoma requiring the expertise of dermatologic and head and neck surgeons, to a patient with advanced Merkel cell carcinoma requiring collaboration between his medical and radiation oncologists. For this very reason, the NCCN Clinical Practice Guidelines in Oncology (NCCN Guidelines) emphasize the importance of multidisciplinary tumor board consultation. Time and time again, however, I am confronted with a reality in which this fundamental and seemingly obvious aspect of oncologic care is not always the case.

Since I trained and have worked for many years at an institution where multidisciplinary care is second nature, it is easy for me to take my colleagues for granted. My patients, though, whatever their diagnosis, remind me on a regular basis how a lack of interdisciplinary collaboration can have disastrous consequences. Simultaneously, ambitious young physicians and colleagues with admirable intentions voice their frustrations about their inability to create an environment in which optimal multidisciplinary care can be delivered.

The value and importance of multidisciplinary care is not a novel concept. Several years ago, a national panel of cancer experts, convened by the NCI, examined how the 6 aims for high-quality care (effective, safe, efficient, equitable, patient-centered, and timely care) proposed by the Institute of Medicine (IOM) applied to oncologic care. ${ }^{1,2}$ In their report, the expert panel considered coordination of care among specialists to be an essential element of delivering high-quality care to patients with cancer.

A central aim identified by the IOM is that care must be effective; that is, based on the best clinical evidence. It is rarely, if ever, possible for one clinician to be fully abreast of the latest developments in all areas of oncology that impact a patient's care. A multidisciplinary team can develop expert consensus guidelines that incorporate the best and most current data across multiple specialties, to provide the most effective care for each patient. The IOM determined that high-quality medical care must be safe and equitable. The NCI expert panel noted that patients with cancer frequently receive inadequate care because established treatment guidelines are not followed. By ensuring that patients receive care that adheres to established best-care guidelines, multidisciplinary clinics can deliver safe, high-quality care in an equitable fashion to all patients.

Another IOM goal for high-quality medical care is efficiency. Studies have shown that multidisciplinary care at a large academic medical center that follows national guidelines can be more cost-efficient than a less organized traditional communitybased approach. ${ }^{3}$ When appropriately structured, the coordinated care provided by multidisciplinary clinics prevents duplication of tests, limits the number of physician visits, and delivers cost-efficient, high-quality care.

The fifth IOM goal is to ensure that care is provided in a patient-centered fashion. Lack of patient education was specifically identified by the NCI as a major barrier to high-quality oncologic care. By providing patients and families with information on their condition and available treatment options, multidisciplinary clinics facilitate

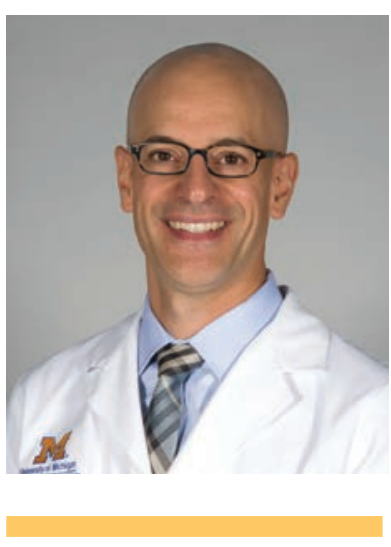

Christopher K. Bichakjian, MD

Dr. Christopher Bichakjian is a Clinical Associate Professor of Dermatology and Associate Chief of the Division of Cutaneous Surgery and Oncology at the University of Michigan (UM). He received his medical degree from Radboud University in The Netherlands. He completed his dermatology residency and fellowship in dermatologic (Mohs) surgery and cutaneous oncology at the UM Health System and Comprehensive Cancer Center. He joined the UM cutaneous surgery and oncology faculty in 2004. Dr. Bichakjian has a special interest in Merkel cell carcinoma and serves as the director of the Comprehensive Cancer Center Multidisciplinary Merkel Cell Carcinoma program which he established in 2006. On a national level, Dr. Bichakjian is heavily involved in staging and clinical guideline development within the field of cutaneous oncology, including melanoma, through the AJCC, NCCN, the American Academy of Dermatology and the American College of Mohs Surgery, and serves as chair of the NCCN Non-Melanoma Skin Cancer Guidelines Panel. 
shared decision-making and provide patients with cancer with a centralized resource to help navigate their care.

Finally, even if all goals are met, the failure to meet them in a timely fashion will undermine the entire process and every best intention. Insufficient teamwork and communication among caregivers frequently results in treatment delays and prevents delivery of high-quality care. A multidisciplinary clinic has the opportunity to provide a single clinical resource that facilitates information transfer among all members of the care team, coordinates appointments, and allows patients to meet with multiple specialists on a single day. In summary, under optimal circumstances, multidisciplinary care overcomes known barriers and embodies each IOM aim for providing high-quality oncologic care.

Why then, despite this knowledge and all of our best intentions, is the ultimate goal so rarely achieved? External explanations are easily identified, which undeniably contribute to the problem. Increasing pressure on clinicians to see more patients and collect more relative value units (RVUs) limits the time physicians can devote to their patients. Documentation requirements, whether for billing purposes, medicolegal protection, or attestation of meaningful use of an electronic health record, further reduce the time doctors can spend caring for patients. Other mundane challenges can arise from the inability to physically bring specialists from different disciplines together if they are spread out across satellites throughout various parts of a metropolitan city or large medical campus. Although modern technology, including video conferencing, can overcome some of these physical hurdles, it cannot replace the communication that occurs when experienced clinicians from multiple specialties gather around a table.

In my opinion, a generous portion of introspection might reveal that the greatest hurdle to be overcome when attempting to achieve optimal oncologic care is the inability or unwillingness of individuals (ie, doctors) to work together. This barrier, if eliminated, would make all other challenges appear trivial. Although teamwork has been instilled in us since early childhood, it sometimes appears that medical school is fraught with a selection bias for the opposite behavior. We all have witnessed examples when egos and personalities have stood in the way of optimal patient care. Disagreement with another person or an entire specialty may prevent an appropriate referral to a more experienced physician.

This practice is unacceptable. Our patients deserve better; our patients deserve the best. Determining how an individual is most appropriately treated is our primary goal as physicians; not who provides the care. One of the most valuable lessons I was taught during my training was to treat my patient as I would want my mother or father to be treated. If physicians could live by this simple motto, teamwork (ie, multidisciplinary care) would come naturally and health care would be elevated to an entirely new level.

\section{References}

1. Aiello Bowles EJ, Tuzzio L, Wiese CJ, et al. Understanding high-quality cancer care: a summary of expert perspectives. Cancer 2008;112:934-942.

2. IOM committee calls for complete revamping of health care system to achieve better quality. Qual Letter Healthcare Leaders 2001;13:14-15.

3. Fader DJ, Wise CG, Normolle DP, Johnson TM. The multidisciplinary melanoma clinic: a cost outcomes analysis of specialty care. J Am Acad Dermatol 1998;38(5 Pt 1):742-751. 